\title{
Urinary tract endometriosis
}

\author{
JEFFREY C. KOSZCZUK, DO \\ MARK FOGLIETTI, DO \\ JOHN F. PEREZ, DO \\ FRANCIS V. DONO, DO \\ ROBERT J. THOMAS, DO
}

\begin{abstract}
Although endometriosis is a common gynecologic pathologic phenomenon, involvement of the urinary tract is relatively rare. The clinical presentation and course of urinary system disease is extremely variable, as illustrated by the seven cases presented in this report. Therapy primarily is surgical, but a thorough understanding of the disease process and a complete knowledge of the patient's history and desires for fertility conservation are necessary to plot the most appropriate treatment course. Bladder involvement is more common, and usually less devastating, than either ureteral or kidney involvement. No signs, symptoms, or physical findings are pathognomonic, and the clinician must maintain a high index of suspicion in all cases of advanced pelvic endometriosis.
\end{abstract}

Although benign, endometriosis has the unique ability to invade tissues and to disseminate, or metastasize, by hematogenous or lymphatic routes or by direct implantation-attributes that essentially are characteristic of malignancies. Endometrial implants and growths have been described in a variety of places, primarily within the pelvis. Ovarian involvement is seen most frequently, but uterine serosa, uterosacral ligaments, the cul-de-sac, fallopian tubes, bladder, rectosigmoid peritoneum, and broad and round ligaments also are commonly involved.

Endometriosis has been encountered with increasing frequency in higher socioeconomic groups. There also is some question as to its association with delayed motherhood. Proposed etiologies include Wolffian or Mullerian duct rests, endometrial migration, serosal metaplasia, retrograde menstruation, direct invasion through uterine wall, transtubal regurgitation, venous, lymphatic, or endometrial metastasis, or surgical transplantation. ${ }^{1}$

The incidence of endometriosis at the operating table is $15 \%$ to $32 \%$. The treatment of choice in many cases is surgery; however, hormonal ther- apy is available for mild to moderate disease (American Fertility Society ${ }^{2}$ stages 1 and 2 [Fig 1]). However, hormonal therapy often is expensive and is unsatisfactory for long treatment periods. Pregnancy may be considered a type of prophylaxis; however, infertility is another significant complication experienced frequently by endometriosis patients.

If there is recurrence following conservative surgical management, additional therapy, including medical hormonal manipulation, further conservative surgery, or even surgical castration, often is necessary.

Endometriosis involving the urinary tract is relatively rare. The spectrum of disease severity varies from incidental findings at laparoscopy, laparotomy, or cystoscopy to more significantly associated backache, hypertension, hematuria, and ureteral or urinary obstruction progressing to nonfunctioning kidney. ${ }^{3}$

We have reviewed seven cases of endometriosis compromising the urinary tract, which were treated by the combined gynecology and urology services at Doctors Hospital in Columbus, Ohio, from 1977 to 1986.

\section{Report of cases}

Of the seven patients reviewed, five were older than 35 years, one was 27 years old, and another was 30 years old. Five patients (cases 1,2,3,5, and 7) were multiparous, one (case 4 ) had had one pregnancy electively terminated, and one (case 6 ) was nulliparous.

These cases illustrate quite well the diagnostic problems that occur in evaluating and treating patients with endometriosis involving the urinary system. Significant clinical data about these cases are presented in Table 1.

\section{Case 1}

The 28-year old patient had had intermittent episodes of cystitis, hematuria, and lower abdominal pain for four years. There were no previous history of endometriosis and no symptoms suggestive of gynecologic disease. She had been treated with several trials of antibiotics for her most recent episode of "hemorrhagic cystitis" without resolution.

A large posterior bladder wall endometrioma was discovered at cystourethroscopic examination. The bladder trigone was not involved. 
Laparotomy was performed, and the endometrioma was excised with a partial bladder resection. The only other pelvic finding was minimal endometriosis of the uterosacral ligaments. Postoperatively, the patient was treated with danazol. Repeat cystourethroscopy six months later was normal.

\section{Case 2}

This 45-year old patient also had intermittent episodes of hemorrhagic cystitis over a prolonged period of time. She also had persistent lower abdominal discomfort and dyspareunia. Conservativemanagement with analgesics and nonsteroidal, anti-inflammatory medications was of little benefit. Pelvic endometriosis had been diagnosed previously, and total abdominal hysterectomy had been performed 14 years earlier.

Cystourethroscopy and biopsy revealedintravesical endometriosisinvolving the bladder trigone. Pelvic laparotomy disclosed no other evidence of pelvic endometriosis. $\mathrm{Bi}$ lateral salpingo-oophorectomy was carried out, and progestogen therapy was begun postoperatively. To date, she is free of the presenting symptomatology and has had no further urologic problems.

\section{Case 3}

A history of profound gross hematuria of three weeks' duration was the presenting complaint of this 46 year-old woman. The patient also had intermittent urinary hesitancy and retention. An episode of acute pyelonephritis, which had occurred four weeks earlier, had been treated with intravenous antibiotics. There was no history of endometriosis.

Nine years prior to this evaluation, total abdominal hysterectomy with bilateral salpingo-ophorectomy had been performed for an unrelated gynecologic problem. She had also been receiving oral estrogen replacement therapy.

Intravenous urography showed evidence of left ureteral obstruction. Less than one-third of left kidney function was demonstrated on the renal scan. Cystourethroscopy had normal findings. Obstruction of the distal left ureter was noted on retrograde ureterograms. A circumferential constriction was identified with left ureteroscopy. The ureteral mucosa appeared grossly normal.
THE AMERICAN FERTILITY SOCIETY REVISED CLASSIFICATION OF ENDOMIETRIOSIS

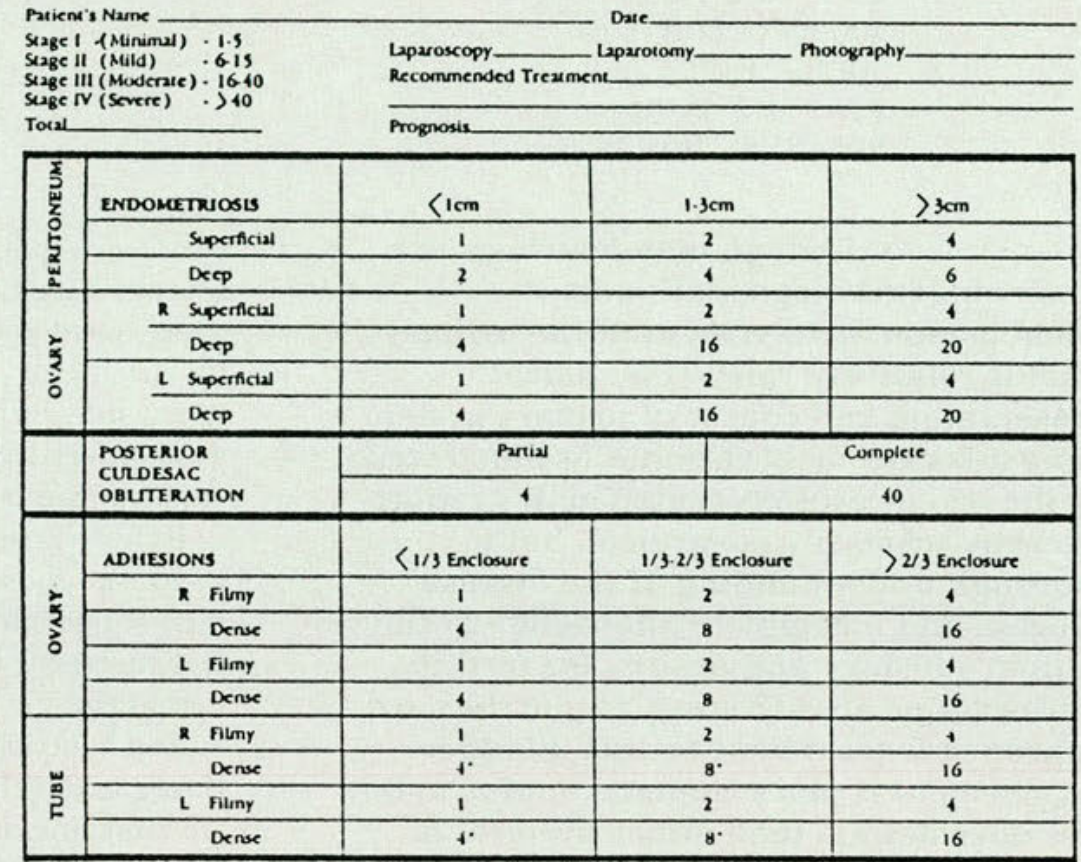

- $W$ the fimbriated end of the tallupian iube us compietely enclosed. change the point assignment to 16

Neditional Endometnosis:

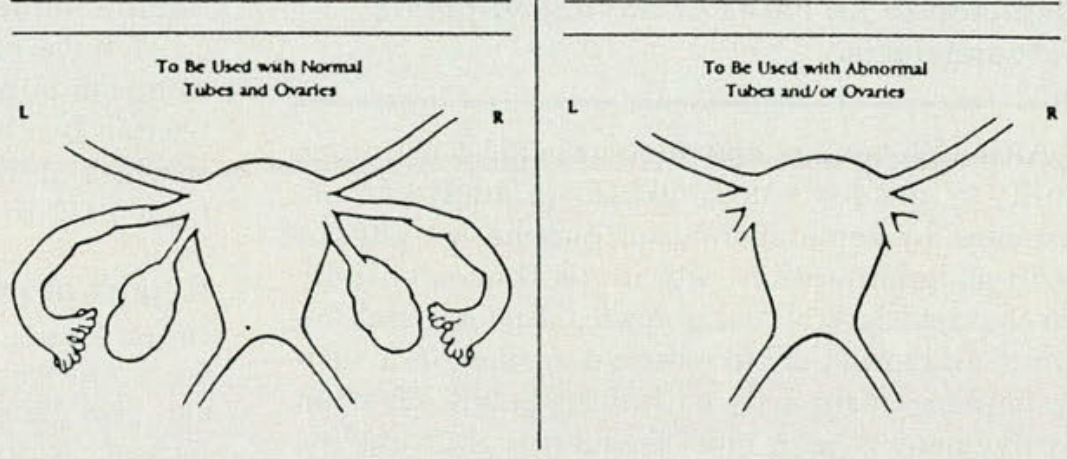

For additional supply write to: The Amerikan Fertillty Soxiety, 2131 Mtagnolia Avenue, Suite 201, Birningham, Alabama 35256

Fig 1. Staging classification (1985 revision) for endometriosis. (Reproduced with permission of the publisiner, the American Fertility Society, Birmingham, Alabama ${ }^{2}$ ).

At laparotomy, a large, encapsulated, but aggressive appearing mass was seen, and malignancy was suspected. Total left nephroureterectomy was performed. No evidence of residual ovarian tissue was noted. Histopathologic examination revealed extensive left ureteral endometriosis with obstruction. Focal invasion of the ureteral wall also was identified. This patient's postoperative recovery was uneventful, and no recurrence has been reported to date.

\section{Case 4}

The 27-year-old patient also had urinary symptoms, in- 

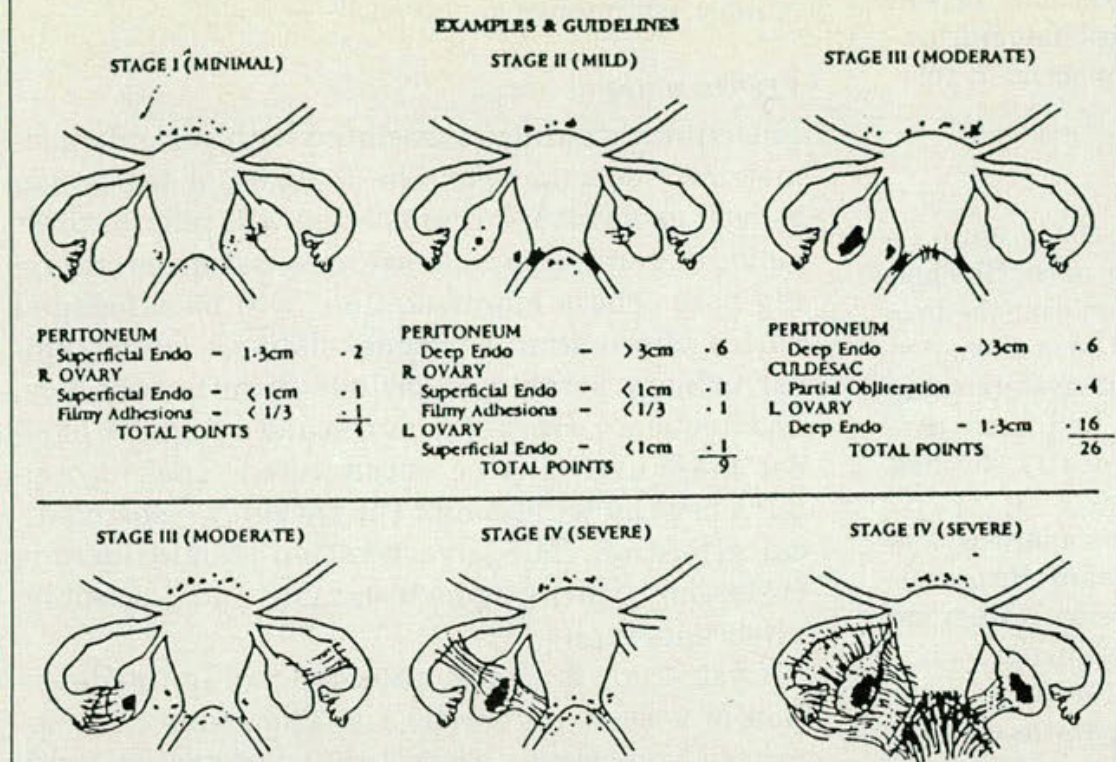

PERITONEUM
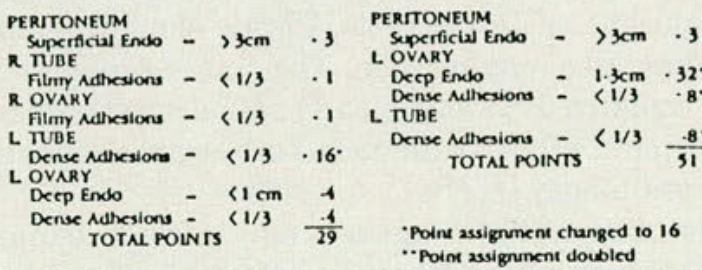

Poind assignment changed to 16 - Potme assignument doubled

Surgical therapy consisted of microsurgical lysis of adhesions and pelvic reconstruction. The right ovary was extensively diseased; therefore, right salpingo-oophorectomy was performed. Danazol therapy was utilized both preoperatively and postoperatively. This patient is now being managed with oral contraceptive therapy, and her symptoms have resolved.

\section{Case 5}

This 43-year-old woman complained of hematuria, dysuria, and urinary urgency. She had had four episodes of cystitis, with outpatient antibiotic therapy, during the previous year. Pelvic endometriosis had been identified with laparoscopy three weeks prior to this admission. An American Fertility Society stage was not assigned, but both ovaries and fallopian tubes were involved. Extensive adnexal adhesions also were noted. Preoperative intravenous urography was normal.

At laparotomy, a large left ovarian endometrioma was seen to invade the left pelvic side wall, with involvement of the left ureter. Left ureteral exploration was necessary to define the course of the left ureter and to remove as much diseased tissue as possible. The uterus, fallopian tubes, and ovaries were removed to complete the procedure.

The postoperative recovery was unremarkable. Current postoperative hormonal management consists of progestogen therapy. Although this case does not illustrate ureteral obstruction, it does show that one must be prepared to proceed with ureteral exploration when extensive extrinsic ureteral

cluding extreme urinary urgency and frequency. In addition, she complained of lower lumbar discomfort radiating to the right anterior thigh, which had persisted for two years. Further symptoms included progressive pelvic pain, dysmenorrhea, and dyspareunia of three years', duration. Stage- 3 pelvic endometriosis had been noted at laparoscopy six months earlier. She was requesting definitive surgical therapy but wished to conserve her fertility.

At cystourethroscopic examination, a posterior bladder wall endometrioma with trigone involvement was identified. disease is encountered.

\section{Case 6}

After one year of danazol therapy, a 30-year-old woman developed dysuria and hematuria. Stage 3 pelvic endometriosis had been diagnosed laparoscopically two years earlier. Response to the danazol had been inadequate.

Cystourethroscopy revealed a large, blue, cystic mass on the posterior bladder wall. The trigone was not involved.

Following pelvic laparotomy, total abdominal hysterectomy with bilateral salpingo-oophorectomy was per- 
formed. A 4-cm posterior bladder wall was excised, and the bladder was partially resected.

There were no postoperative complications. Repeat cystourethroscopy was normal six months later, and lowdose, cyclic estrogen and progestogen replacement therapy was begun.

\section{Case 7}

The 38-year-old patient was admitted to the hospital by her family doctor with a suspected pelvic mass. She had had persistent right lower abdominal pain over the previous six months. Pyelonephritis also had been diagnosed and treated recently. A total abdominal hysterectomy with bilateral salpingo-oophorectomy had been performed three years earlier, and subsequently she had been given estrogen replacement therapy.

Right ureteral obstruction with hydronephrosis was noted on intravenous urography. Cystrourethroscopically, the bladder appeared normal. However, retrograde ureterograms showed distal ureteral obstruction.

At laparotomy, the path of the right ureter was explored, and a distal right periureteral mass was excised. Distal right ureterectomy with ureteroneocystostomy was performed. No evidence of residual ovarian tissue was found. Histopathologic examination revealed extrinsic ureteral endometriosis. Postoperative recovery was uneventful. Only mild, residual right ureteropyeloectasis was identified with intravenous urography six months postoperatively.

\section{Discussion}

The cases presented here demonstrate that surgery is of primary importance in the treatment of endometriosis with urinary tract involvement. Medical therapy, primarily danazol, is used adjunctively. However, the reversal of ureteral obstruction secondary to endometriosis has been reported after primary therapy with danazol ${ }^{4,5}$ and progestin. ${ }^{6}$ It must be remembered that the resolution or remission of endometriosis with hormonal therapy alone usually will be maintained only as long as the medication is continued.

It has been reported ${ }^{7}$ that cure rates, as determined by the percentage of conceptions and pregnancies following therapy, are similar for women with mild to moderate disease who were treated either medically (with danazol) or with conservative, pelvic reconstructive surgical techniques. However, it must be emphasized that danazol, like other forms of medical therapy, cannot be considered definitive treatment for endometriosis. Patients with moderate to severe disease often will require surgical intervention after medical therapy has been deemed inadequate or intolerable, or after it has been discontinued. ${ }^{8}$

Cullen ${ }^{9}$ first described urinary tract endometriosis in 1918 in a 27-year-old woman with advanced adenomyoma of the rectovaginal septum. Both ure- ters were involved. Bladder involvement is more common than ureteral disease, while the kidney is least commonly involved. ${ }^{10,11}$

\section{Vesical endometriosis}

The clinical findings associated with vesical endometriosis depend upon the location of the lesion as well as its size. Patients often will refer to their pelvic discomfort as a pressure sensation or cramping pain. These manifestations will be associated with cyclic ovarian hormonal changes. Other typical urinary symptoms include dysuria, urgency, and frequency. Gross hematuria and a palpable bladder mass also may be encountered. These symptoms usually will prompt the patient to seek medical attention. The investigation should include cystoscopy, intravenous urography, and, possibly, ultrasonography. ${ }^{12}$

Cystoscopy is a most valuable tool in the detection of vesical endometriosis. The lesion may appear to be markedly congested and edematous, with translucent or bluish cysts. Tissue sloughing and bleeding also may be seen. The tumor probably is best visualized cystoscopically at the onset of menstruation. Definitive diagnosis is by cystoscopically directed biopsy. ${ }^{11,13,14}$

The differential diagnosis may include benign diseases (varices, angioma, papilloma, localized vesical inflammation, or ulceration) as well as malignant diseases (infiltrative or metastatic vesical carcinoma). ${ }^{11}$

The management plan for vesical endometriosis must take into account the individual desires of a well-informed patient. Age, family planning desires, extent of disease, and severity of symptoms are important variables to consider. Therefore, there is a spectrum of possible therapies, which range from conservative hormonal management with progestins or danazol, to conservative excision and reconstructive surgical procedures (partial cystectomy or pelvic microsurgery, or both), to surgical excision and castration. Conservative surgical procedures offer the best results for relatively young patients desiring future pregnancies. ${ }^{11,13}$

\section{Ureteral endometriosis}

Endometriosis involving the ureter is less common than that involving the bladder and has an associated greater morbidity. Ureteral disease can lead to crippling pain, with ureteral obstruction and hydronephrosis progressing to complete loss of kidney function. ${ }^{15-18}$ In case 3 , the patient lost more than two-thirds of her left renal function. Conversely, pelvic endometriosis may completely obstruct the ureters and destroy the kidneys with few 


\begin{tabular}{|c|c|c|c|c|}
\hline Case & Age (yr) & Year & Presentation & Disposition \\
\hline 1 & 28 & 1983 & $\begin{array}{l}\text { Hematuria and dysuria } \\
\text { Intermittent urinary } \\
\text { tract infections } \\
\text { No history of endometriosis } \\
\text { No gynecologic symptoms } \\
\text { Vesical endometriosis }\end{array}$ & $\begin{array}{l}\text { Cystoscopy with bx } \\
\text { Laparotomy with } \\
\text { partial cystectomy } \\
\text { Postoperative danazol }\end{array}$ \\
\hline 2 & 45 & 1984 & $\begin{array}{l}\text { Endometriosis (diagnosed } \\
\text { in 1962) } \\
\text { Total abdominal hysterectomy } \\
\text { (1968) }\end{array}$ & $\begin{array}{l}\text { Cystoscopy with bx } \\
\text { Laparotomy with lateral } \\
\text { salpingo-oophorectomy } \\
\text { Postoperative progestoger }\end{array}$ \\
\hline
\end{tabular}

Hematuria, dysuria, and cystitis

Lower abdominal pain

Dyspareunia

Vesical endometriosis

3
Total abdominal hysterectomy and bilateral salpingooophorectomy (1976)

Dysuria and hematuria

Urinary hesitancy and retention

Pyelonephritis

Left ureteral obstruction

Less than 1/3 left renal function

Estrogen replacement

Intrinsic ureteral endometriosis

Frequency and urgency

Pelvic pain, dysmenorrhea, and dyspareunia

Stage 3 endometriosis

Vesical endometriosis

Dysuria, hematuria and urgency

Urinary tract infections,

Pelvic endometriosis

Extrinsic left ureteral endometriosis

Danazol therapy (one year)

Vesical endometriosis

Abnormal uterine bleeding

Pelvic and back pain, dyspareunia

Hematuria and dysuria

Stage 3 endometriosis (1984)

Vesical endometriosis

Total abdominal

hysterectomy and

bilateral salpingo-

oophorectomy (1974)

Estrogen replacement (started 1974)

Possible pelvic mass

Pyelonephritis

Right lower quadrant pain

Ureteral obstruction with hydronephrosis

Extrinsic ureteral endometriosis
Cystoscopy (normal)

Retrograde ureterogram (abnormal)

Total left nephroureterectomy

Cystoscopy with bx

Microsurgical pelvic reconstruction with right salpingo-oophorectomy

Preoperative and postoperative danazol

Laparotomy, total abdominal hysterectomy with bilateral salpingo-oophorectomy

Cystoscopy with bx

Laparotomy, total abdominal hysterectomy, bilateral salpingo-oophorectomy

Partial cystectomy

Postoperative estrogen and progestogen

Cystoscopy (normal)

Retrograde ureterography (abnormal)

Laparotomy

Right ureteral exploration

Excision of periureteral mass

Ureteroneocystostomy 
or no symptoms. ${ }^{18}$

Ureteral endometriosis is classified either as intrinsic or extrinsic. With intrinsic disease, invasion of the ureteral wall occurs. It is this form of disease that may be mistaken for a primary ureteral neoplasm, thus resulting in nephroureterectomy as primary surgical therapy. Again, case 3 exemplifies this situation.

With extrinsic ureteral endometriosis, there is no invasion beyond the adventitia, and the ureter is compressed externally. The attendant external inflammation and fibrosis also may lead to ureteral scarring and obstruction. ${ }^{17-20}$ Cases 5 and 7 illustrate extrinsic disease.

Symptoms of ureteral endometriosis most commonly include abdominal or flank pain, or both. Gross and microscopic hematuria are common with intrinsic disease. ${ }^{18,21}$ Dysmenorrhea, dyspareunia, dysuria, and urgency or frequency also are common. Radiologic investigation often will reveal evidence of ureteral obstruction, hydronephrosis, and nonfunctioning kidneys. ${ }^{21}$

The diagnosis of ureteral involvement usually is made during surgery. Treatment must be based on the status of the involved as well as the uninvolved kidney, and on whether obstruction is present. If a kidney is severely damaged, nephrectomy is the procedure of choice. If the kidney is salvageable, the strictured area must be resected and properly reanastomosed.

The patient's desires regarding the possible conservation of ovarian and reproductive function also are important in formulating the surgical treatment plan. If retention of reproductive capacity is desired, local excision must be as complete as possible, and the surgery may be more in the province of the urologist than the gynecologist. With extensive disease in which a kidney is nonfunctioning, surgical castration should be recommended, as well as excision of all diseased tissues. ${ }^{18,22,23}$

\section{Renal endometriosis}

Endometriosis of the kidney itself, although extremely rare, has been reported. ${ }^{24,25}$ A patient with kidney involvement will present with flank pain, swelling, and hematuria. Various degrees of renal deformity, hydronephrosis, and renal failure have been encountered. The pathogenesis probably involves embryonic deposits of Mullerian ducts.

\section{Postoperative medical management}

Recurrent endometriosis after surgical castration has been reported, and controversy exists over the use of estrogen replacement therapy after surgical castration. ${ }^{26-28}$ Indeed, recurrent endometriosis with urinary tract involvement also has been re- ported ${ }^{14,29}$ in postmenopausal women. The patients in cases 3 and 7 had recurrent endometriosis with ureteral obstruction years after surgical castration. They had been receiving estrogen replacement therapy.

These isolated cases represent interesting and perplexing problems and, fortunately, are uncommon. A young woman who has undergone surgical castration because of severe endometriosis probably should not be deprived of the protection that appropriate cyclic estrogen and progestin therapy provides against the symptoms of the climacteric/ menopause syndrome and the devastations of osteoporosis.

If the amount of disease was extensive, or if residual endometriosis remains after surgical castration, it is wise to use progrestogen rather than estrogen therapy for the first six postoperative months. This should prevent reactivation of disease in most cases. Estrogen replacement therapy may then be instituted at as low a dose as possible $(0.625 \mathrm{mg}$. to $1.25 \mathrm{mg}$. of conjugated estrogens daily). ${ }^{23,30-32}$

\section{Conclusions}

Of the seven cases presented in this report, only two were staged in accordance with the American Fertility Society classification system. Because of the highly variable clinical manifestations of endometriosis, this system is very important to achieve some order in the correlation of therapeutic results with severity of disease. It becomes invaluable when evaluating and reporting these results in the medical literature. Therefore, the staging classification presented in Fig 1 should be used for all patients with endometriosis when they are laparoscopically and surgically evaluated or treated.

The clinician is challenged with the problem or early diagnosis of urinary tract endometriosis. Although signs and symptoms, along with physical findings, may be helpful, there is nothing pathognomonic about the presentation of urinary system involvement. A high index of suspicion must be maintained, and intravenous urography is recommended in all cases of advanced pelvic endometriosis that require surgical intervention.

1. Kane C, Drouin P: Obstructive uropathy associated with endometriosis. Am J Obstet Gynecol 1985;151:207-211.

2. American Fertility Society: Revised American Fertility Society classification of endometriosis. Fertil Steril 1985;43:351-352.

3. Moore JG, Hibbard LT, Growdon WA, et al: Urinary tract endometriosis: Enigmas in diagnosis and management. Am J Obstet Gynecol 1979;134:162-172.

4. Gardner B, Whitaker RH: The use of danazol for ureteral obstruction caused by endometriosis. $J$ Urol 1981;125:117-118. 
5. Rivlin ME, Krueger RP, Wiser WL: Danazol in the management of ureteral obstruction secondary to endometriosis. Fertil Steril 1985;44:274276.

6. Gantt PA, Hunt JB, McDonough PG: Progestin reversal of ureteral endometriosis. Obstet Gynecol 1981;57:665-67.

7. Guzick DS, Rock JA: A comparison of danazol and conservative surgery for the treatment of infertility due to mild or moderate endometriosis. Fertil Steril 1983;40:580-584.

8. Kitchin JD III: Endometriosis, in Sciarra JJ (ed): Gynecology and Obstetrics. Philadelphia, J.B. Lippincott Co, 1986.

9. Cullen TS: Adenomyoma of the rectovaginal septum. Bull Johns Hopkins Hosp 1918;28:343-349.

10. Skor AB: Warren MM, Mueller EO: Endometriosis of the bladder. Urology 1977;9:689-692.

11. Arap NW, Lopes RN, Cury M, et al: Vesical endometriosis. Urology $1984 ; 24: 271-274$.

12. Goodman JD, Macchia RJ, Macasaet MA, et al: Endometriosis of the urinary bladder: Sonographic findings. AJR 1980;135:625-626.

13. Aldridge KW, Burns JR, Singh B: Vesical endometriosis: A review of 2 case reports. $J$ Urol $1985 ; 134: 539-541$.

14. Vorstman B, Lynne C, Politano VA: Postmenopausal vesical endometriosis. Urology 1983;22:540-542.

15. Kerr WS Jr: Endometriosis involving the urinary tract. Clin Obstet Gynecol 1966;9:331-357.

16. Slutsky JN, Callahan D: Endometriosis of the ureter can present as renal failure: A case report and review of endometriosis affecting the ureters. J Urol 1983;130:336-337.

17. Klein RS, Cattolica EV: Ureteral endometriosis. Urology 1979;13:477482.

18. Reddy AN, Evans AT: Endometriosis of the ureters. $J$ Urol $1974 ; 111: 474-480$

19. Rising JA, Hassen HB, Obstructive ureteral endometriosis: A case report $J$ Urol 1967;98:77-80.

20. Stanley KE Jr, Utz DC, Dockerty NB: Clinically significant endometriosis of the urinary tract. Surg Gynecol Obstet 1965;120:491-498.
21. Stiehm WD, Becker JA, Weiss RM: Ureteral endometriosis. Radiology 1972;102:563-564.

22. Smith T: The surgical management of endometriosis. Clin Obstet Gynecol 1978;5:557-

23. Mattingly RF, Thompson JD: TeLinde's Operative Gynecology, ed 6. Philadelphia, J.B. Lippincott Co, 1985, pp 257-286.

24. Marshall VF: The occurrence of endometrial tissue in the kidney: Case report and discussion. $J$ Urol 1943;50:652-656.

25. Bazaz-Malik G, Saraf V, Rana BS: Endometrioma of the kidney: Case report. J Urol 1980;123:422-423.

26. Ocshner T, Markland C: Endometriosis obstructing the ureter. J Urol $1967 ; 98: 462-465$.

27. Andrews WC, Larsen GD: Endometriosis: Treatment with hormonal pseudo pregnancy and/or operation. Am J Obstet Gynecol 1974;118:643651.

28. Ranney B: The prevention, inhibition, palliation, and treatment of endometriosis. Am J Obstet Gynecol 1975;123:778-785.

29. Stewart WW, Ireland GW: Vesical endometriosis in a postmenopausal woman: A case report. $J$ Urol 1977;118;480-481.

30. Mishell DR, Brenner PF: Management of Common Problems in Obstetrics and Gynecology. Oradell, NJ, Medical Economics Books, 1983, pp 434-438.

31. Speroff L, Glass RH, Kase NG: Clinical Gynecologic Endocrinology and Infertility, ed 3. Baltimore, Williams and Wilkins Co, 1982, pp 463469.

32. Langmade CF: Pelvic endometriosis and ureteral obstruction. Am J Obstet Gynecol 1975;122:463-469.

From the Departments of Obstetrics and Gynecology and General Surgery and Urology, Doctors Hospital North, Columbus, Ohio.

Reprint requests to Dr Perez, $100 \mathrm{~W}$. Third Avenue, Columbus, $\mathrm{OH} 43025$. 